APRECIACIÓN, EVALUACIÓN

Y DESACUERDOS ESTÉTICOS

- Eleonora Orlando . 


\section{Eleonora Orlando}

Universidad de Buenos Aires, IIF (SADAF-CONICET)

\section{Apreciación, evaluación y desacuerdos estéticos}

DOI: $10.36446 /$ be.2021.57.268

\section{Resumen}

La tesis principal del artículo es que los predicados estéticos densos, tales como 'perturbador', 'sombrío, 'equilibrado', son sensibles a la apreciación, en el mismo sentido en que lo son los predicados de gusto, $y$, por lo tanto, pueden ser analizados en el marco del relativismo de la verdad. La razón principal que se aduce en favor de esta tesis es que se trata de predicados cuyo uso requiere la aplicación de un estándar estético. Se distinguen dos tipos: por un lado, los predicados experienciales involucran estándares constituidos por estados psicológicos contingentes y aleatorios, característicos de perspectivas estéticas idiosincráticas; por otro, los predicados teóricos involucran la adopción de estándares estables, determinados por perspectivas estéticas canónicas. Esta distinción es utilizada para explicar diferencias entre unos y otros: el uso de predicados experienciales puede dar lugar a desacuerdos estéticos sin falta, semejantes a los desacuerdos sin falta generados por los predicados de gusto, pero eso no ocurre con los predicados teóricos.

\section{Palabras clave}

Predicado estético denso; Sensibilidad a la apreciación; Relativismo de la verdad; Perspectiva estética; Desacuerdo sin falta

\section{Aesthetic Assessment, Evaluation and Disagreement}

\section{Abstract}

The aim of this paper is to propose that thick aesthetic predicates, such as 'shocking', 'sombre' and 'balanced', can be considered to be assessment-sensitive, in the sense in which predicates of personal taste have been thought to be, and hence be analysed within the framework of truth relativism. The main reason offered in support of this thesis is that their use requires the application of an aesthetic standard. Moreover, a distinction between experiential and theoretical aesthetic predicates is introduced: the former involve standards that are constituted by contingent and random psychological states and, hence, characteristic of idiosyncratic aesthetic perspectives, whereas the latter rely on the adoption of stable ones, determined by canonical aesthetic perspectives. This distinction is used to account for differences between one another: experiential predicates feature in faultless disagreements, as is the case with predicates of personal taste, while this is not the case with theoretical ones.

\section{Keywords}

Thick aesthetic predicate; Assessment-sensitivity; Truth relativism; Aesthetic perspective; Faultless disagreement

Recibido: 11/08/21. Aprobado: 10/09/21.

Palabras como 'equilibrado', 'sombrío', 'perturbador', 'armonioso', 'dinámico', 'poderoso', 'vívido', 'sentimental', 'delicado', 'trágico', 'trillado', 'estridente' constituyen predicados estéticos, basados en los que Sibley (1959) llamó 'conceptos estéticos'. Son un tipo de predicados densos, es decir, predicados que no son puramente descriptivos, como 'alto', sino que contienen un componente evaluativo. Dan lugar a enunciados estéticos densos, como (1) y (2)

(1) Judith decapitando a Holofernes es perturbadora.

(2) Mujer joven leyendo una carta es equilibrada.

Estos enunciados son evaluativos porque sus predicados lo son: en general, aplicar 'perturbadora' a una obra de arte implica no sólo atribuirle cierto rasgo sino también expresar una actitud evaluativa global (positiva o negativa) hacia ese rasgo $-o$, más exactamente, hacia la posesión de ese rasgo por parte de la obra. En este ensayo, me propongo analizar qué tipo de predicados son los predicados estéticos densos y, como consecuencia de ello, a qué tipos de desacuerdo pueden dar lugar enunciados como (1) y (2); en otras palabras, qué variedades de desacuerdo puede involucrar el juicio estético.

La estructura del trabajo es la siguiente. En la primera sección, caracterizo un tipo de desacuerdo, el desacuerdo sin falta, que suele generarse en relación con el uso de predicados de gusto, e introduzco la noción de sensibilidad a la apreciación que ha sido propuesta por los filósofos del lenguaje para explicar ese fenómeno (MacFarlane 2007, 
2014). En la segunda sección, presento cuatro ejemplos distintos de desacuerdos estéticos. A continuación, en la tercera sección, propongo una explicación del funcionamiento semántico de los predicados estéticos densos que los asimila a los predicados de gusto: se trata, en ambos casos, de predicados sensibles a la apreciación. La propuesta incluye de manera central una distinción entre predicados estéticos experienciales y predicados estéticos teóricos. Los predicados experienciales dependen del desarrollo de perspectivas estéticas idiosincráticas, dominadas por estándares contingentes y aleatorios, mientras que los predicados teóricos dependen de la adopción de perspectivas estéticas canónicas, estabilizadas en torno a un canon artístico. En la cuarta sección, analizo los desacuerdos iniciales en términos de esta distinción; como consecuencia de ello, sostengo que sólo pueden considerarse sin falta aquellos desacuerdos estéticos que involucran predicados experienciales. La conclusión resume las tesis principales defendidas en el artículo.

\section{1. ¿QUÉ ES UN DESACUERDO SIN FALTA?}

Los predicados estéticos antes mencionados se parecen a los predicados de gusto, tales como 'delicioso', 'elegante', 'divertido': tanto unos como otros son términos densos, es decir, tienen un componente evaluativo. Al emitir un enunciado de gusto como (3)

(3) Las aceitunas griegas son deliciosas.

no sólo se atribuye una propiedad a las aceitunas sino que además se expresa una evaluación positiva, a diferencia de lo que ocurre al emi$\operatorname{tir}(4)$

(4) Las aceitunas griegas se venden en el mercado naturista. en donde la aplicación del predicado, puramente descriptivo, 'se venden en el mercado naturista' no involucra necesariamente la expresión de una actitud evaluativa de ningún signo. En las últimas dos décadas, los filósofos del lenguaje han prestado especial atención a ciertos desacuerdos que surgen en relación con enunciados como (3), tal como puede verse en este diálogo:

\section{Desacuerdo sobre gustos}

Ana: Las aceitunas griegas son deliciosas. No pueden compararse con las comunes.

Pedro: No coincido. No me gusta la textura que tienen. Yo prefiero las comunes.

Si bien Ana y Pedro expresan opiniones opuestas sobre las aceitunas griegas, no diríamos que alguno de ellos está cometiendo un error, como sí lo haríamos si la discusión fuera en cambio la siguiente:

\section{Desacuerdo fáctico}

Ana: Las aceitunas griegas se venden en el mercado naturista. Pedro: No, no es cierto. No se venden en ningún negocio del barrio.

En este último caso la existencia de opiniones opuestas sobre un mismo asunto implica que o bien Ana o bien Pedro está cometiendo un error: sólo uno de esos enunciados es verdadero porque es un hecho que alguien vende en el barrio aceitunas griegas o es un hecho que nadie lo hace. En síntesis, mientras que este desacuerdo involucra un error o una falta por parte de uno de los participantes, el primero revela un desacuerdo sin falta.

Más específicamente, si bien Ana puede defender a muerte a las aceitunas griegas y creer que Pedro está cometiendo un grave error, no 
es el mismo tipo de error anterior: no se trata de cometer un error acerca de cómo es el mundo sino de no compartir cierto gusto, tener otro estándar de gusto, o, incluso, no pertenecer al mismo mundo cultural o "ver el mundo de otra manera". Es decir, estrictamente hablando, Pedro no está equivocado sino que es distinto de Ana, tiene otras preferencias, o anhela tener otras experiencias sensibles. Por eso se suele decir que ese tipo de desacuerdo es un desacuerdo sin error o falta, y como tal, persiste, a menos que cambie no la condición epistémica sino algo más profundo y ligado a las emociones, algo que podría llamarse 'el perfil subjetivo' de una persona, constituido por sus gustos y preferencias. El viejo dicho "De gustibus non est dispuntandum" expresa, en mi opinión, no que sobre gustos no hay discusiones (porque es obvio que las hay) sino que las discusiones sobre gustos son peculiares, dado que no tienen por objeto la identificación de una única posición correcta sino la transformación del otro, el cambio de su perfil subjetivo, su adopción de nuevas pautas culturales; no son discusiones en el sentido de 'conversaciones en las que se ofrecen razones en favor de una única posición correcta'.

Durante las últimas dos décadas, los filósofos del lenguaje propusieron entonces una explicación relativista de la diferencia entre los predicados de gusto y los puramente descriptivos, que está relacionada con los distintos tipos de desacuerdo que originan. Desde ese marco teórico, conocido como 'relativismo de la verdad' (fundamentalmente MacFarlane 2007, 2014; también Lasershon 2005), los predicados de gusto son considerados sensibles a la apreciación, y es esta propiedad la que permite dar cuenta de los desacuerdos sin falta. Más específicamente, los predicados de gusto ('delicioso', 'elegante', 'divertido'), a diferencia de los puramente descriptivos que expresan propiedades fácticas ('griega', 'en venta en el mercado naturista'), son sensibles al punto de vista del apreciador o juez. Esto determina que un uso de una oración como (3) varíe de valor de verdad en función de quién sea la persona que aprecie o juzgue esa emisión: independientemente de que en su contexto original, que, supongamos, tuvo a Ana como hablante, resultara verdadera, puede resultar falsa si es apreciada o juzgada por otra persona, como Pedro, por ejemplo, cuyas preferencias y gustos son distintos de los suyos. En términos más técnicos, cada uso de (3) debe ser evaluado en relación con el estándar de gusto del contexto de apreciación, es decir, el estándar de gusto de la persona que lo aprecia o juzga. Por lo tanto, con respecto al primer ejemplo de diálogo, Desacuerdo sobre gustos, la emisión de Ana es una afirmación que resulta verdadera en relación con su contexto de apreciación, dominado por el estándar de gusto de Ana, mientras que la negación de esa afirmación por parte de Pedro resulta verdadera en relación con su contexto de apreciación, que es distinto del de Ana, dado que involucra un estándar de gusto diferente. Si se toma en cuenta que hay distintos estándares de gusto en juego, resulta que ninguno de ellos está equivocado. Pero, por supuesto, desde la perspectiva de cada uno, dominada por el propio estándar, lo que el otro dice es falso.

En síntesis, mientras que las afirmaciones fácticas se evalúan como verdaderas o falsas con respecto a los hechos del mundo, las afirmaciones de gusto se evalúan como verdaderas o falsas no sólo con respecto a los hechos del mundo sino además en relación con un estándar de gusto, el propio del apreciador, es decir, el provisto por el contexto de apreciación. Esta es la manera relativista de reflejar la idea de que los desacuerdos y las discusiones acerca de cuestiones de gustos no tienen el propósito de eliminar un error objetivo e identificar una única posición correcta: en el nuevo marco propuesto, su propósito podría describirse como el de eliminar un error e identificar la posición correcta relativamente a la adopción de cierta perspectiva, es decir, desde el marco constituido por ciertos gustos y preferencias, $o$, en otros términos, cierto mundo cultural. 
El concepto de sensibilidad a la apreciación permite entonces considerar que los usos de oraciones como (3) son afirmaciones y, por tanto, pueden ser verdaderos o falsos. En este punto el relativismo de la verdad se distingue del expresivismo, heredero del emotivismo ético de Stevenson (1944), según el cual los enunciados de gusto no son afirmaciones ( $\mathrm{y}$, por tanto, no pueden ser verdaderos o falsos) $\mathrm{y}$ los predicados que incluyen poseen un contenido puramente expresivo determinado por la expresión de preferencias subjetivas.

\section{EJEMPLOS DE DESACUERDO ESTÉTICO}

El mismo contraste anterior entre predicados densos y puramente descriptivos se presenta en el ámbito de la estética. Mientras que (1) y (2) contienen predicados densos, los predicados en (5) y (6) son puramente descriptivos:

(5) Judith decapitando a Holofernes está en Florencia.

(6) Mujer joven leyendo una carta pertenece al Barroco Holandés.

Aplicar a una obra predicados que expresan propiedades fácticas, como estar en Florencia o pertenecer al Barroco Holandés, no implica evaluarla - a menos que se trate de un contexto particular, en donde por razones sociales, históricas o culturales, ciertas propiedades fácticas estén sistemáticamente asociadas con algún tipo de evaluación. ${ }^{1}$ Ahora bien, si bien puede haber discusiones acerca de si $J u$ dith decapitando a Holofernes está en Florencia o en Washington

${ }^{1}$ Un predicado como 'estar en la Galleria degli Uffizi' sería un ejemplo de un predicado puramente descriptivo sistemática pero no convencionalmente asociado con una evaluación positiva: es porque la Galleria degli Uffizi es reconocida como un museo prestigioso en nuestra cultura que el predicado adquiere una connotación positiva, pero si el contexto cultural cambiara dejaría de tenerla.
D.C. o si Mujer joven leyendo una carta pertenece al Barroco Holandés o al Renacimiento tardío, las discusiones propiamente estéticas suelen involucrar enunciados como (1) y (2) y no como (5) y (6).

Veamos entonces, en primer lugar, el siguiente diálogo:

\section{Desacuerdo 1}

Ana: Judith decapitando a Holofernes es perturbadora. Prefiero la representación más idealizada del tema en el cuadro de Botticelli.

Isabel: Sí, claro que es perturbadora. Ahí está el secreto de su poder y su atractivo.

Ana e Isabel coinciden en aplicar el predicado 'perturbadora' a la obra de Gentileschi, pero desacuerdan acerca de la evaluación que ese rasgo conlleva: para Ana ser perturbadora es algo negativo (un efecto desagradable de la escena de violencia que nos muestra), mientras que para Isabel constituye un mérito (dado que es el rasgo que la hace poderosa y atractiva). Veamos ahora este otro ejemplo:

\section{Desacuerdo 2}

Ana: Judith decapitando a Holofernes es perturbadora. Prefiero la representación más idealizada del tema en el cuadro de Botticelli.

Julia: No, no es perturbadora. Tiene su cuota de violencia pero representa sin duda la fuerza y la decisión de dos mujeres valientes.

Acá el desacuerdo es de otro tipo: Ana y Julia desacuerdan respecto de la aplicación misma del predicado 'perturbadora' a la obra de Gentileschi - aunque bien podrían acordar en que ser perturbadora en un rasgo negativo del cuadro. 


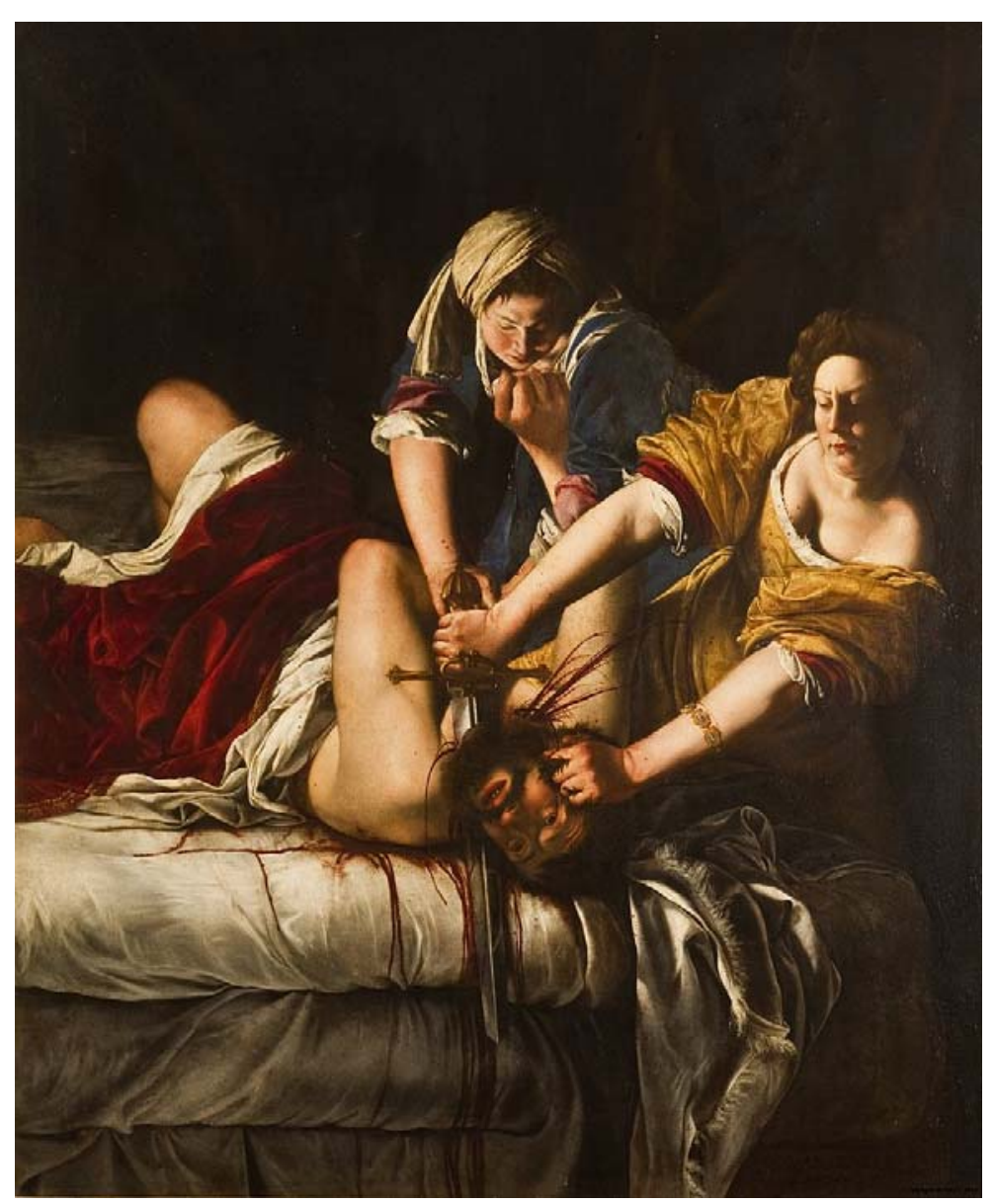

Artemisia Gentileschi, Judith decapitando a Holofernes, 1620-1621. Galería de los Oficios, Florencia.
Estas discusiones conducen a preguntarse por el tipo de desacuerdo que se revela en cada caso. ¿Qué significa desacordar acerca de la aplicación de un predicado estético a una obra de arte? ¿Implica desacordar respecto de una evaluación, respecto de una propiedad o respecto de ambas cosas? ¿Cómo se soluciona el desacuerdo? ¿Hay alguien que está equivocado, o puede considerarse en cambio un desacuerdo sin falta, es decir, un desacuerdo en el que dos personas sostienen posiciones opuestas sobre un tema pero ambas son correctas? En términos del primer ejemplo, ¿es posible que Ana no esté equivocada al evaluar negativamente el carácter perturbador del cuadro de Gentileschi y, al mismo tiempo, que Isabel tampoco lo esté al evaluarlo positivamente? En términos del segundo ejemplo, ¿es posible que Ana no esté equivocada al considerar que la obra es perturbadora y, al mismo tiempo, que Julia tampoco lo esté al considerar que no lo es? En síntesis, ¿son los desacuerdos estéticos asimilables a los desacuerdos acerca de gustos, presentados en la sección anterior?

Otra duda relevante es si la respuesta que se dé a estas preguntas puede generalizarse de manera de comprender a todos los predicados estéticos, en particular, a predicados como el que aparece en (2). Imaginemos los siguientes diálogos:

\section{Desacuerdo 3}

Pedro: Mujer joven leyendo una carta es equilibrada, como todas las pinturas de Vermeer. Eso contribuye a que sea perfecta. Simón: Sí, es verdad que es equilibrada. Pero eso es justamente lo que no me atrae de la obra.

¿Es posible que Pedro considere que ser equilibrada sea un rasgo positivo del cuadro de Vermeer mientras que Simón lo considere negativo, pero, sin embargo, ninguno esté come-tiendo un error? 


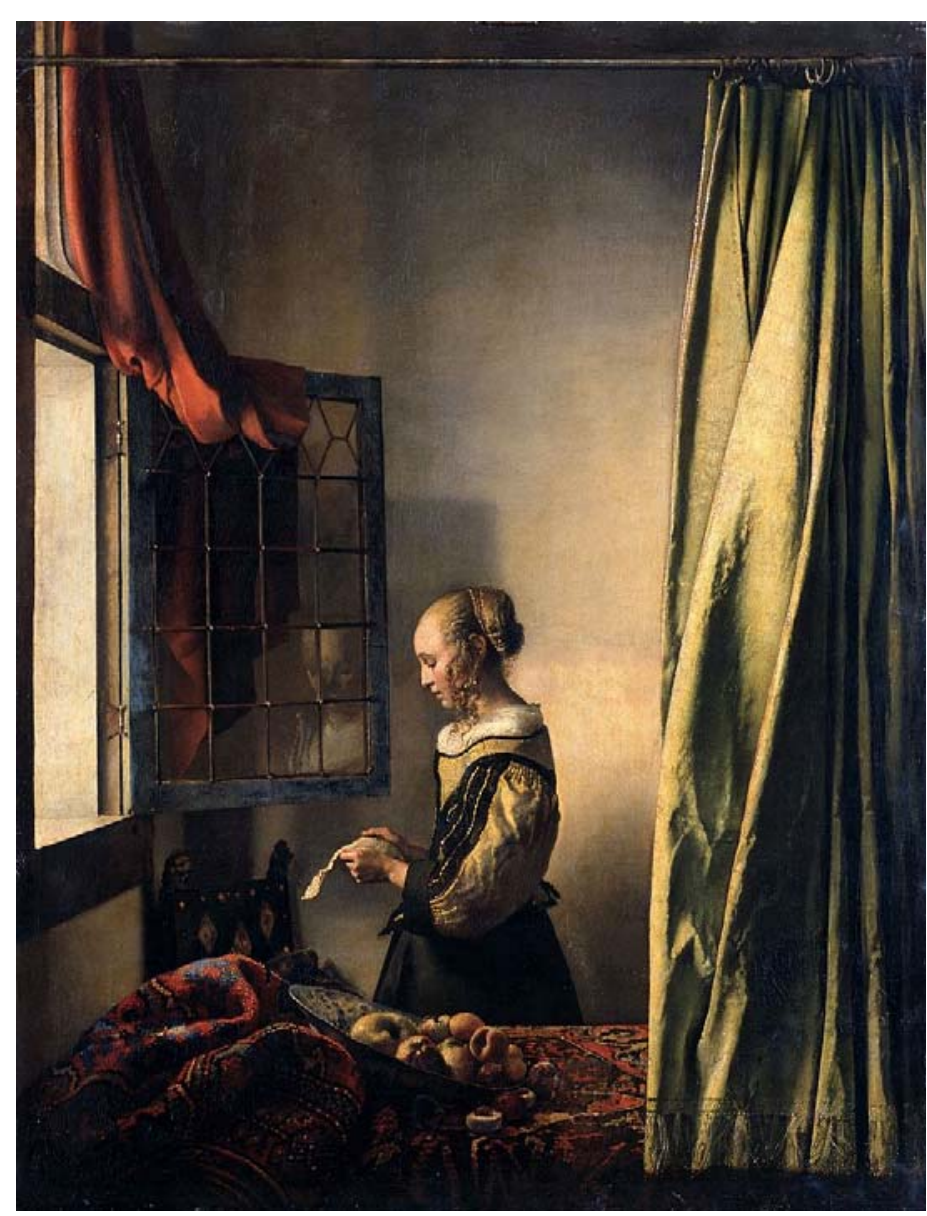

Johannes Vermeer, Mujer joven leyendo una carta, 1657-1659. Galería de los Maestros Antiguos, Dresde.
Por último, ¿qué intuiciones despierta este otro ejemplo?

\section{Desacuerdo 4}

Pedro: Mujer joven leyendo una carta es equilibrada.

Tomás: No, no estoy de acuerdo. No es lo que yo llamaría 'una obra equilibrada'.

¿Es posible que Pedro y Tomás desacuerden respecto de si la obra de Vermeer es equilibrada y, sin embargo, que las dos posiciones sean correctas, es decir, que tampoco en este caso alguien esté cometiendo un error? Más específicamente, ¿puede tanto afirmarse con verdad que el cuadro de Vermeer es equilibrado como negarse, con verdad, que lo sea?

En la sección siguiente, ofreceré una explicación del funcionamiento semántico de los predicados estéticos densos en el marco del relativismo de la verdad, que permitirá entender qué tipo de desacuerdo ejemplifican estos diálogos, en particular, si puede o no considerarse que revelan desacuerdos sin falta.

\section{UNA EXPLICACIÓN RELATIVISTA DE LOS PREDICADOS ESTÉTICOS DENSOS}

\subsection{Escalaridad, multidimensionalidad y sensibilidad a la apreciación}

Los predicados estéticos densos pertenecen al conjunto de los adjetivos gradables. La gradabilidad es una propiedad sintáctica que distingue a ciertos adjetivos que se caracterizan por admitir modificadores de grado ('muy equilibrada', 'un poco sombrío', 'demasiado perturbadora'), y figurar en construcciones comparativas ('más equilibrada que', 'menos sombrío que') y ecuativas ('tan perturbadora como'). 
La cara semántica de la gradabilidad es la escalaridad: un adjetivo gradable representa una propiedad escalar. Este tipo de propiedad se aplica a un objeto no de manera absoluta sino en un cierto grado, lo cual involucra que ha alcanzado un umbral en una escala - es decir, el objeto se "mapea" en un grado de una escala que coincide o supera cierto umbral. En términos de un ejemplo, 'alto', un adjetivo gradable puramente descriptivo, se aplica a un individuo determinado si este alcanza cierto umbral, contextualmente determinado, en una escala de altura: bajo la hipótesis de que Pedro mide $1,80 \mathrm{~m}$, se le aplicará 'alto' si lo que está en discusión es la altura media de los chicos de quince años y el umbral es fijado, por ejemplo, en 1,70 m, mientras que si se está haciendo referencia a posibles candidatos para un equipo de básquet y el umbral es 2 metros, el adjetivo no se le aplicará en ese contexto. En general, la semántica de un adjetivo gradable requiere la determinación de un umbral en una escala, lo cual involucra la apelación a una clase de comparación contextualmente determinada (como en el ejemplo anterior, la clase de los chicos de quince años o la clase de los jugadores de básquet) o algún otro tipo de criterio que permita establecer a partir de qué grado de la escala correspondiente el objeto va a contar como poseyendo cierta propiedad (en el ejemplo anterior, como siendo alto). ${ }^{2}$

La multidimensionalidad expresa el hecho de que un predicado estético denso se aplica a un objeto no sobre la base de un solo aspecto (como la altura) sino a partir de la consideración conjunta de diversos aspectos. ${ }^{3}$ Más específicamente, es posible considerar que a una obra de arte se le asigna un grado de una escala en función de una

${ }^{2}$ El locus classicus para un tratamiento formal específico de este tipo de adjetivos es Kennedy (2007). Véase también Kennedy \& McNally (2005).

${ }^{3} \mathrm{La}$ tesis de que los predicados estéticos densos son multidimensionales ha sido también defendida, mediante un argumento distinto, en Stojanovic (2016) y Stojanovic \& McNally (2017). combinación de distintas dimensiones suyas que son individualmente seleccionadas y medidas, y luego comparativamente ponderadas. La aplicación del predicado 'sombrío' a una pintura, por ejemplo, involucra seleccionar ciertos aspectos que se consideran individualmente relevantes (tales como los colores, las formas, las figuras, el tema), establecer un umbral de carácter sombrío para cada uno de esos aspectos (un color sombrío no es lo mismo que un tema sombrío), y luego determinar cuál es la importancia comparativa de cada uno de ellos, es decir, cuál es el peso que tiene cada uno de esos aspectos en el conjunto total (alguien puede considerar que lo que hace sombrío a un cuadro son fundamentalmente sus colores, mientras que otra persona puede pensar que lo que más influye en la determinación de su carácter sombrío es el tema al que alude). Es sobre la base de combinar diversos aspectos (seleccionados, medidos y ponderados) que puede fijarse un umbral en la escala correspondiente a 'sombrío', y determinarse si una determinada obra alcanza o no ese umbral. El punto principal que quiero destacar es que la aplicación de un predicado estético presupone entonces la posesión de un estándar estético, es decir, un criterio que guía los procedimientos de selección, medición y ponderación de distintos aspectos de una obra. ${ }^{4}$

Esto remite a la otra característica fundamental que es posible atribuir a los predicados estéticos, a saber, la sensibilidad a la aprecia-

${ }^{4}$ Es pertinente aclarar que estos procedimientos no son realizados de manera consciente por el hablante competente sino que se trata de destrezas involucradas en la competencia lingüística con los predicados estéticos; de la misma manera, ser competente en el uso de 'alto' no involucra ser consciente de que cada vez que se usa el predicado se está fijando contextualmente un umbral en la escala de altura, o, por lo menos, no implica que el hablante sea capaz de describir su competencia lingüística en esos términos. 
ción. Es precisamente porque la aplicación de estos predicados involucra la consideración conjunta de diversas dimensiones de una obra, lo cual requiere disponer de un estándar estético, por lo que pueden considerarse sensibles a la apreciación, en el sentido definido para los predicados de gusto. Así como la verdad o falsedad de un uso de (3) depende de cuál sea el estándar de gusto del apreciador o juez, la verdad o falsedad de los usos de (1) y (2), repetidas abajo,

(1) Judith decapitando a Holofernes es perturbadora.

y

(2) Mujer joven leyendo una carta es equilibrada.

dependerá de cuál sea en cada caso el estándar estético del apreciador o juez.

Ahora bien, como se destacó en la sección anterior, los predicados estéticos son no sólo descriptivos sino también evaluativos, es decir, su uso está asociado con una evaluación por parte del hablante. Intuitivamente, al producir y al apreciar enunciados como (1) y (2), el hablante no sólo está representándose algo desde cierta perspectiva sino que también está evaluando esa representación perspectival: en términos del primer ejemplo, al afirmar que Judith decapitando a Holofernes es perturbadora, parte de lo que el hablante está haciendo es transmitir una evaluación de cierto aspecto de la obra de Gentileschi (en particular, su potencial perturbador).

Hay distintas maneras de explicar esta estrecha conexión entre el predicado y la evaluación. Mi propuesta es que todo predicado denso, además de estar semánticamente correlacionado con una propiedad multidimensional, involucra otro factor semántico, independiente del anterior, de carácter evaluativo. Este factor es un valor global (po- sitivo o negativo) codificado por el predicado, sistemáticamente correlacionado con una actitud evaluativa del mismo signo por parte del hablante competente. En la línea de la tradición metaética, llamaré 'valencia' a ese valor global lingüísticamente codificado. ${ }^{5}$

Es importante tomar en cuenta que la valencia es no sólo conceptualmente diferente sino también independiente de la propiedad multidimensional: determinar que Mujer joven leyendo una carta alcanza el grado $n$ de equilibrio (el umbral) en un determinado contexto es un proceso independiente del de evaluar, en ese contexto, la posesión del rasgo como algo (estéticamente) bueno o malo, es decir, como un mérito o demérito (estético) de la obra. La valencia del predicado estético puede ser entonces considerada un significado expresivo de tipo evaluativo, independiente de la propiedad multidimensional, que sería su significado descriptivo o representacional.

De este modo, el primer componente semántico, la propiedad multidimensional, contribuye a determinar las condiciones de verdad de los distintos usos de oraciones en las que aparece el predicado; el segundo componente, la valencia, es, en cambio, un componente semántico que determina las condiciones de corrección evaluativa de esos usos. Como resultado de ello, los usos de enunciados estéticos no sólo tienen un valor de verdad (pueden ser verdaderos o falsos)

${ }^{5}$ El reconocimiento de este componente transforma a esta propuesta en una versión de expresivismo (o no-cognitivismo) metaestético que puede ser comparada con algunas versiones del expresivismo (o no-cognitivismo) metaético, según el cual los conceptos éticos densos expresan en parte actitudes de aprobación y desaprobación. La propuesta original es, como mencioné, la de Stevenson (1944). Versiones posteriores se encuentran en Gibbard (1990) y Blackburn (1992). Como señalan Hurka \& Elstein (2009), el componente evaluativo global también puede ser concebido en términos cognitivistas, i.e., en términos de los conceptos BUENO y MALO. (Uso mayúsculas, como es usual, para designar conceptos.) 
sino que también tienen una carga evaluativa o valencia (pueden tener valencia positiva o negativa). ${ }^{6}$

De acuerdo con lo dicho, la valencia del enunciado es fijada por la actitud evaluativa del hablante, pero me interesa destacar que esta depende, al igual que la fijación del umbral en la escala multidimensional, de la posesión de un determinado estándar estético, es decir, de la perspectiva estética que adopte el apreciador o juez. En otros términos, el componente expresivo-evaluativo del predicado estético es tan sensible a la apreciación como el componente descriptivo-representacional. Tanto la selección y la ponderación de los aspectos relevantes de una obra como la evaluación global subsiguiente requieren la posesión de un estándar estético que funcione como guía, y ese estándar es, en cada caso, el utilizado por quien aprecia o juzga los enunciados estéticos en juego. De este modo, los juicios estéticos resultan tanto verdaderos o falsos como evaluativamente correctos o incorrectos no de manera absoluta sino en relación con la perspectiva estética determinante de ese estándar. Es decir, las condiciones en las que los usos de (1) y (2) son verdaderos y evaluativamente correctos (es decir, su valencia es adecuada) dependerán del estándar estético del apreciador o juez. ${ }^{7}$

Como argumentaré a continuación, considero que hay dos tipos de predicados estéticos, los experienciales y los teóricos. De este modo,

\footnotetext{
${ }^{6} \mathrm{Si}$ bien por cuestiones de espacio no me ocuparé de desarrollar en detalle este aspecto, la propuesta implica analizar a los predicados estéticos densos en términos de una semántica dualista, análoga a las que se han aplicado a otros tipos de términos que poseen una dimensión evaluativa, como, por ejemplo, los peyorativos de grupo. Véanse, por ejemplo, las teorías de McCready (2010) y Predelli (2013), inspiradas en Potts (2005) y, en última instancia, en Kaplan (1999).

${ }^{7}$ De este modo, un juicio estético sería estrictamente un enunciado estético consi-
} derado desde la perspectiva de un juez (o apreciador). la variabilidad en el valor de verdad y en la valencia de un enunciado estético dependerá de cómo pueda caracterizarse el predicado involucrado.

\subsection{Los predicados experienciales}

Los predicados como 'perturbador' y otros semejantes ('conmovedor', 'provocativo', etc.) derivan de verbos de experimentante ('perturbar', 'conmover', 'provocar'), es decir, verbos psicológicos que proyectan un argumento de experimentante. Algo es perturbador porque perturbó a alguien, a un sujeto $x$, donde este es el argumento de experimentante del verbo 'perturbar' -en este caso, el experimentante es el objeto directo del verbo. ${ }^{8}$ El adjetivo derivado también proyecta un argumento de experimentante, en este caso oculto o implícito. ${ }^{9}$ De acuerdo con esto, una obra es perturbadora/conmovedora/provocativa para alguien, es decir, en función del efecto variable que tiene en el sujeto de la experiencia estética, por lo general, alguien con un determinado perfil emocional - como resultado de su experiencia estética, el sujeto resulta perturbado, conmovido, provocado. Propongo llamar a estos predicados estéticos que son un subconjunto de los predicados psicológicos 'predicados estéticos experienciales'.

\footnotetext{
${ }^{8}$ Los verbos psicológicos se clasifican en tres grupos, según la función sintáctica desempeñada por el argumento de experimentante: verbos con sujeto experimentante ('amar'), verbos con objeto directo experimentante ('perturbar') y verbos con objeto indirecto experimentante ('gustar'). Agradezco este dato a Andrés Saab.

${ }^{9}$ Véanse Glanzberg $(2007,2009)$ y Schafer (2011), quienes señalan este aspecto en relación con adjetivos de gusto personal como 'delicioso' ('tasty') y 'divertido' ('fun’). Véase también McNally \& Stojanovic (2017), para una defensa del mismo punto de vista.
} 
Tomaré como ejemplo de predicado experiencial al adjetivo que aparece en (1), 'perturbadora'. El potencial perturbador de una obra es un rasgo constituido por el estado psicológico de ser o estar perturbado, un tipo de estado emocional con una connotación negativa (cuya naturaleza precisa excede los límites de este trabajo). Lo que me interesa destacar es que se trata de una propiedad estética constitutivamente vinculada con un estado psicológico que puede variar en función de la sensibilidad y las creencias del sujeto, las cuales pueden ser individuales o estar socialmente determinadas. Estos estados mentales concomitantes influyen entonces aleatoriamente en su experiencia estética. Ahora bien, de acuerdo con lo explicado anteriormente, la aplicación del predicado a una obra involucra, por un lado, una selección y ponderación de distintas dimensiones, y por otro, una evaluación positiva o negativa de esa condición. Dado que en este caso esos procedimientos involucran un estado psicológico que puede variar aleatoriamente de acuerdo con el sujeto de la experiencia estética, el estándar involucrado es un estándar estético idiosincrático (el cual, como sugerí antes, puede ser individual o estar socialmente determinado).

Es importante enfatizar que es su dependencia respecto de estándares idiosincrásicos lo que permite dar cuenta de la variabilidad que caracteriza a las valencias de los predicados experienciales. De acuerdo con el tipo de valencia involucrada, los predicados estéticos densos han sido clasificados en dos grupos (Väyrynen 2011; Kirchin 2013, 2017; McNally \& Stojanovic 2017). Por un lado, están aquellos que tienen una valencia específica, ya sea positiva o negativa, tales como 'equilibrado' (+), 'armonioso' (+), 'estridente' (-). Por otro, están aquellos cuyas valencias no están especificadas o definidas como positivas o negativas sino que pueden resultar de una manera u otra según el contexto, tales como 'provocativo' $( \pm)$, 'perturbador' $( \pm)$, 'vívido' $( \pm) .{ }^{10}$ Ahora bien, todos los predicados experienciales tienen valencias no específicas o indefinidas, que se especifican o definen en un contexto determinado.

En términos de nuestro primer ejemplo, presupongamos que, para decidir si 'perturbadora' se aplica o no al cuadro de Gentileschi, las dimensiones relevantes que deben tomarse en cuenta son los colores utilizados, las expresiones de los personajes y la acción representada. Presupongamos, además, que Ana es muy sensible a la violencia, es decir, que le generan un gran rechazo las escenas de violencia como destaqué, esta sensibilidad bien puede ser producto de una educación en la que a las mujeres se les enseña, desde chicas, a rechazar la violencia; de este modo, la sensibilidad de una persona suele ser algo socialmente determinado o construido, es decir, algo que no es puramente individual sino parte de un perfil social. Como consecuencia, Ana da mayor peso a la cruenta acción atribuida a Judith y su doncella que a las otras dimensiones del cuadro. Esto hace que Ana resulte perturbada por el cuadro de Gentileschi, es decir, desde su perspectiva, el predicado 'perturbadora' se aplica a la obra. Del mismo modo, es decir, también en función de su estándar estético idiosincrático, Ana asigna al predicado una valencia negativa, en tanto considera que ser perturbadora no representa un valor estético para el cuadro sino que se trata de una característica que le quita valor, en comparación, por ejemplo, con el cuadro de Botticelli sobre el mismo tema.

Por lo tanto, un uso de (1), nuevamente repetida abajo,

(1) Judith decapitando a Holofernes es perturbadora. ${ }^{10}$ Represento a la valencia del predicado mediante los signos '+' , '-' o ambos (cuando
no está definida). 
puede ser tanto verdadero como falso y puede tener tanto una valencia positiva como negativa, en función del estándar estético idiosincrático del sujeto de la experiencia. Este primer tipo de estándar puede entonces caracterizarse como un criterio para seleccionar, medir y ponderar aspectos de una obra así como para evaluarlos positiva o negativamente, que está basado en un estado psicológico del sujeto de la experiencia que puede cambiar aleatoriamente en función de otros estados concomitantes (como, por ejemplo, cierta sensibilidad a la violencia, socialmente determinada).

En síntesis, la sensibilidad a la apreciación de los predicados estéticos experienciales puede ser entendida en términos de su dependencia respecto de la posesión de un estándar estético idiosincrático. De este modo, una afirmación de (1) será verdadera o falsa y tendrá una valencia positiva o negativa en relación con un estándar estético idiosincrático; en otros términos, en relación con lo que MacFarlane llama 'un contexto de apreciación', constituido en este caso por lo que puede denominarse 'una perspectiva estética idiosincrática'.

\subsection{Los predicados teóricos}

Predicados estéticos tales como 'equilibrado', 'dinámico', 'armonioso', en cambio, no son adjetivos que derivan de verbos de experimentante $y$, por lo tanto, no puede considerarse que posean un argumento de experimentante oculto o implícito; es decir, no son predicados psicológicos. Representan propiedades formales de una obra, aquellas que están asociadas con rasgos estructurales y temáticos que son independientes de los efectos variables de la obra en el sujeto de la experiencia. En otras palabras, son propiedades que no están constitutivamente vinculadas con estados psicológicos que pueden variar en función de la sensibilidad y las creencias del sujeto, es decir, de estados mentales concomitantes que influyen aleatoriamente en su experiencia estética - aun cuando se trate, en muchos casos, de sensibilidades y creencias socialmente determinadas. Estas propiedades formales se corresponden con conceptos definidos en el marco de ciertas corrientes y escuelas artísticas. Si bien los conceptos estéticos están claramente influenciados por características sociales, políticas y culturales de las sociedades y momentos históricos a los que pertenecen las respectivas corrientes y escuelas, la aplicación de estos predicados no depende de efectos psicológicos aleatorios que pueda tener la obra en el sujeto de la experiencia estética: propongo, por tanto, llamarlos 'teóricos'. Una obra es equilibrada, dinámica, armoniosa en virtud de cumplir con ciertas condiciones impuestas por la definición de equilibrio, dinamismo, armonía en el marco de ciertas tradiciones o escuelas. Por lo tanto, es plausible pensar que estos predicados dependen de los estándares estéticos canónicos representados por dichas corrientes y escuelas artísticas.

Es importante destacar que las definiciones de conceptos estéticos como los de equilibrio, dinamismo, armonía y semejantes por lo general no son claras y explícitas para los artistas en el momento de la creación de sus obras, y tampoco suelen serlo para los apreciadores en el momento en que interactúan con ellas. ${ }^{11}$ Sí lo son, en cambio, para los críticos e historiadores del arte, que son quienes están en condiciones de justificar tanto la verdad (o la falsedad) como la corrección (o incorrección) evaluativa de los casos particulares de (2), $\mathrm{y}$, en general, de los juicios estéticos con predicados teóricos.

Para explicar este punto, me centraré en el ejemplo (2), repetido abajo,

(2) Mujer joven leyendo una carta es equilibrada.

\footnotetext{
${ }^{11}$ Agradezco a Diana Pérez por haber hecho que tomara conciencia de este aspecto.
} 
Por un lado, la verdad o falsedad de todo uso de este enunciado depende de la presencia en la obra de Vermeer de ciertos rasgos que ejemplifican un concepto estético, el equilibrio (visual), en el marco de un movimiento artístico, el Barroco Holandés. Si bien ese concepto se define en función de cierto efecto en la psicología humana, una sensación de reposo y estabilidad, producida por el hecho de que nuestra atención está distribuida equitativamente entre los elementos percibidos (no hay tensión producida por la percepción de alguno de ellos en particular), se trata de un efecto genérico y no particular del sujeto que tiene la experiencia estética en función de su sensibilidad o sus creencias (las cuales, como vimos, pueden ser tanto individuales como socialmente determinadas). La obra es equilibrada porque ejemplifica el concepto de equilibrio en el Barroco Holandés, no porque produce un efecto psicológico en el sujeto de la experiencia estética que puede variar aleatoriamente en función de estados mentales concomitantes; en consecuencia, la obra es equilibrada de acuerdo con un estándar estético canónico, que determina la aplicación del concepto de equilibro definido en el marco del Barroco Holandés.

Por otro lado, el estándar estético característico de una perspectiva canónica suele determinar el respaldo de las valencias específicas que tienen los predicados teóricos en el marco de los respectivos cánones artísticos. ${ }^{12}$ De este modo, todo uso (evaluativamente) correcto de (2)

\footnotetext{
${ }^{12}$ Sin embargo, es importante observar que las valencias definidas o específicas también son variables, en el sentido de que no siempre deben ser respaldadas sino que pueden ser revertidas. En mi propuesta, esto puede explicarse en los siguientes términos: la adopción de una perspectiva estética canónica determina la reversión de la valencia específica de un predicado teórico porque el concepto correspondiente es redefinido en el marco de la corriente artística que la origina. Un ejemplo podría ser el uso de "El carnaval de Juanito es estridente (-)" cuando es juzgada o apreciada por un sujeto que adopta la perspectiva canónica del Kitsch contemporáneo, en
}

tendrá una valencia positiva, como consecuencia del respaldo de la valencia positiva de 'equilibrada', también determinada por la perspectiva canónica del Barroco Holandés.

En síntesis, la sensibilidad a la apreciación de los predicados estéticos teóricos puede ser entendida en términos de su dependencia respecto de la adopción de un estándar estético canónico. Dado que ese estándar es establecido por los críticos e historiadores del arte, y, por lo general, es ignorado por los apreciadores, el uso de estos predicados suele ser un uso deferencial, esto es, involucra una deferencia a la comunidad de expertos, los cuales introducen los términos en el lenguaje y conocen con precisión sus respectivas condiciones de aplicación. Una afirmación de (2) será entonces verdadera o falsa y su valencia será positiva o negativa en relación con un estándar estético canónico; es decir, en relación con lo que MacFarlane llama 'un contexto de apreciación', constituido en este caso por lo que puede denominarse 'una perspectiva estética canónica'. ${ }^{13}$

En consecuencia, cada tipo de perspectiva estética -idiosincrática o canónica- determina un rango distinto de variabilidad en el valor

cuyo marco la valencia negativa de 'estridente' es revertida y, por tanto, el uso posee una valencia positiva.

${ }^{13}$ Vale la pena destacar que algunos predicados teóricos representan propiedades formales de las obras que están en parte constituidas por reacciones perceptivas, tales como 'vívido' y 'estridente', entre otros. Una pintura es vívida en parte porque sus rasgos (trazos, figuras, colores, sonidos) suelen impactar fuertemente en las facultades perceptivas del sujeto; lo mismo puede decirse de una obra estridente: no sólo tiene ciertos rasgos particulares sino que además impacta fuertemente de manera sistemática en el aparato perceptivo de quien tiene la experiencia. Sin embargo, considero que este aspecto no los vuelve experienciales, en el sentido señalado, puesto que no se trata de reacciones subjetivas individuales, dependientes de estados psicológicos concomitantes que influyen aleatoriamente en la experiencia estética del sujeto. 
de verdad y en la valencia del juicio estético. Más específicamente, el rango de variabilidad determinado por las perspectivas estéticas idiosincráticas es mayor que el determinado por las perspectivas canónicas. En consecuencia, los casos de desacuerdos que involucran predicados experienciales, dependientes de perspectivas idiosincráticas, son distintos de los casos que involucran predicados teóricos, dependientes de perspectivas canónicas.

\section{ANÁLISIS DE LOS DESACUERDOS ESTÉTICOS}

4.1 Desacuerdos con predicados experienciales

Comenzaré por analizar Desacuerdo 2, repetido abajo por razones expositivas:

\section{Desacuerdo 2}

Ana: Judith decapitando a Holofernes es perturbadora. Prefiero la representación más idealizada del tema en el cuadro de Botticelli.

Julia: No, no es perturbadora. Tiene su cuota de violencia pero representa sin duda la fuerza y la decisión de dos mujeres valientes.

Es posible suponer que tanto Ana como Julia coinciden en que los aspectos relevantes a tomar en cuenta para la aplicación del adjetivo 'perturbadora' a una obra son sus colores, las expresiones y actitudes de sus personajes, y la acción representada (en caso de que los haya, como ocurre en el ejemplo). Asimismo, como sugerí anteriormente, debido a su sensibilidad a la violencia, podríamos imaginar que a Ana le genera un profundo rechazo cualquier escena que involucre la decapitación de una persona; Julia, en cambio, profundamente consciente de los prejuicios que influyen en la educación de las mujeres, siente una profunda admiración por la iconografía feminista de Gentileschi. El desacuerdo entre ambas puede explicarse entonces en términos como los siguientes. Ana considera que los colores y las expresiones son poco perturbadores pero la acción, dado su rechazo de la violencia, lo es en grado extremo; Julia, por su parte, acuerda con Ana en que los colores y las expresiones son poco perturbadores, $y$ también en que la acción es perturbadora. Pero, mientras que Ana le da mucha importancia a la cruenta escena representada, Julia, concentrada en los caracteres y las expresiones de los personajes, la interpreta como una mera excusa para mostrar el carácter fuerte de Judith y su doncella, y su capacidad para defenderse, con valentía inusitada, del peligro que representan ciertos hombres (es decir, pone a la violencia en un segundo plano). Como resultado, Ana ubica a la obra por encima del umbral que debe alcanzar una pintura para resultar perturbadora, mientras que Julia lo hace por debajo de ese umbral.

En síntesis, si bien Ana y Julia seleccionan los mismos aspectos como relevantes y le asignan a cada uno el mismo grado de potencial perturbador, no coinciden en su importancia comparativa: para Ana la acción, con su carga de violencia, tiene el mayor peso, mientras que Julia considera que la expresión controlada, decidida y concentrada de las mujeres es el aspecto más significativo. En consecuencia, Ana, extremadamente sensible a la violencia y, por tanto, fuertemente impactada por la decapitación, encuentra a la obra perturbadora, mientras que Julia, satisfecha con el retrato de la fuerza femenina, niega que lo sea, a pesar de la acción violenta. Se trata de un desacuerdo sin falta porque Ana y Julia utilizan en cada caso sus propios estándares estéticos idiosincráticos, asociados con el predicado experiencial 'perturbadora', influenciados, respectivamente, por el fuerte disgusto generado por la representación de la violencia y la aplicación de categorías feministas en la interpretación de la historia del arte. 
En cuanto a Desacuerdo 1, recordemos que la sensibilidad a la apreciación afecta no sólo al componente descriptivo-representacional sino también al componente expresivo-evaluativo, lo que antes llamé 'valencia'. De este modo, la aplicación de un estándar estético involucra no sólo la selección y medición de aspectos y su ponderación comparativa sino además la evaluación global de lo que implica para una obra poseer cierta propiedad multidimensional. La posibilidad de coincidir en el primer conjunto de procedimientos pero no en la evaluación global puede tomarse entonces como evidencia de que el predicado posee dos componentes semánticos distintos e independientes entre sí. Esto implica que en el caso del uso de un predicado experiencial como 'perturbadora', los estándares idiosincráticos involucrados por dos sujetos distintos pueden determinar la misma selección, medición y ponderación de aspectos pero distinta evaluación global. Eso es exactamente lo que ejemplifica Desacuerdo 1, también reproducido abajo:

\section{Desacuerdo 1}

Ana: Judith decapitando a Holofernes es perturbadora. Prefiero la representación más idealizada del tema en el cuadro de Botticelli.

Isabel: Sí, claro que es perturbadora. Ahí está el secreto de su poder y su atractivo.

Por un lado, Ana e Isabel, coinciden en considerar perturbadora a la obra de Gentileschi (bajo la hipótesis de que ambas reconocen los mismos aspectos como relevantes, y le asignan no sólo idéntico potencial perturbador sino también idéntico peso a cada aspecto). Por otro, cada una evalúa el potencial perturbador de la obra de manera opuesta: según Ana, es algo que le quita mérito o valor estético, mientras que para Isabel constituye un mérito o valor estético de la obra.
Nuevamente, se trata de un desacuerdo sin falta porque tanto Ana como Isabel evalúan el potencial perturbador de la obra de Gentileschi en función de sus respectivos estándares estéticos idiosincráticos, lo cual las conduce a especificar la valencia del predicado de manera opuesta: nuevamente, es posible presuponer que Ana, dada su educación tradicional, detesta la representación de la violencia, lo que la lleva a evaluar negativamente a los cuadros que encuentra perturbadores, mientras que Isabel, tal vez también inspirada por la interpretación feminista de la historia del arte, considera que el potencial perturbador de una obra es un rasgo positivo.

\subsection{Desacuerdos con predicados teóricos}

Analizaré ambos casos de manera conjunta. Nuevamente, invierto el orden de los diálogos:

\section{Desacuerdo 4}

Pedro: Mujer joven leyendo una carta es equilibrada.

Tomás: No, no estoy de acuerdo. No es lo que yo llamaría 'una obra equilibrada'.

\section{Desacuerdo 3}

Pedro: Mujer joven leyendo una carta es equilibrada, como todas las pinturas de Vermeer. Eso contribuye a que sea perfecta. Simón: Sí, es verdad que es equilibrada. Pero eso es justamente lo que no me atrae de la obra.

En Desacuerdo 4, Pedro y Tomás desacuerdan en aplicar el adjetivo 'equilibrada' a la obra de Vermeer; en Desacuerdo 3, mientras que Pedro acuerda con Simón en considerarla equilibrada, Pedro le atribuye a ese rasgo un valor positivo mientras que Simón lo considera un rasgo negativo de la obra. Ahora bien, cuando Pedro afirma (2) 
no está utilizando un criterio idiosincrático para seleccionar, medir y ponderar distintos aspectos de la obra, así como evaluar globalmente esos aspectos, sino que está aplicando un concepto estético que es parte del canon artístico del Barroco Holandés - como es plausible presuponer, sobre la base de su deferencia a los expertos. Más específicamente, es posible presuponer que las dimensiones relevantes a tomar en cuenta para caracterizar a una pintura como equilibrada serían la posición de los objetos en el plano, la paleta de colores y distribución de la luz, y la jerarquía de tamaños. Nótese que, a diferencia de lo que ocurre con el carácter sombrío y el potencial perturbador, en este caso no es posible asociar una escala de equilibrio con cada uno de los aspectos porque el equilibrio de la obra no depende del equilibrio de sus diferentes aspectos sino que son otras propiedades de esos aspectos los que determinan el equilibrio global. El equilibrio se define como una propiedad que resulta de la combinación de esos aspectos, no como una propiedad que pueda pertenecerles a ellos de manera independiente. En cuanto al primer aspecto mencionado, la posición de los objetos, cabe destacar que la figura de la mujer ocupa un lugar central, acompañada por una ventana y una cortina abiertas. En relación con la distribución de la luz, tanto su cara como la carta que está leyendo aparecen iluminadas (y la luz se refleja suavemente en la pared, la cortina y la tela y los frutos que están sobre la mesa, lo cual se manifiesta a través de una delicada difuminación de los colores). Finalmente, en cuanto a los tamaños comparativos de los objetos, la mujer se ve pequeña en comparación con la ventana y la cortina: su pequeñez es compensada, sin embargo, por la centralidad de su figura. La pequeñez de la mujer puede indicar que está pasivamente a merced de las noticias que recibe; nótese que hay una doble revelación: por un lado, material, dado que la carta es leída gracias a la luz que entra por la ventana y, por otro, mental, puesto que la mujer parece enterarse de algo importante a través de su lectura. La disposición de los objetos en el plano, la distribución temática y la gradualidad en el manejo de la luz y los colores, conjuntamente con la proporcionalidad de los tamaños permiten considerar al cuadro de Vermeer como un ejemplo de equilibrio bajo una expresión típica de la pintura barroca holandesa.

De este modo, la afirmación de Pedro es verdadera y tiene la valencia correcta (positiva) en relación con un estándar estético canónico, que constituye el respectivo contexto de apreciación. Las negaciones de Tomás y de Simón involucran entonces contextos de apreciación desde los cuales se impugna esa perspectiva canónica - en el caso de Tomás, se rechaza la ubicación de la obra por sobre el umbral de equilibrio, mientras que en el caso de Simón se invierte el valor positivo tradicionalmente atribuido al equilibrio.

En mi opinión, las réplicas deben interpretarse como el resultado de la aplicación de un concepto estético distinto, es decir, de un concepto que no es el concepto canónico de equilibrio: al negar (2), Tomás y Simón no están utilizando un criterio idiosincrático de selección, medición, ponderación y evaluación global, pero tampoco están aplicando el concepto de equilibrio reconocido por el canon del Barroco Holandés sino que están introduciendo un nuevo concepto, cuya definición deberá hacerse explícita en la discusión. Probablemente se trate de un concepto semejante, por ejemplo, de la manera como el concepto de equilibrio se redefine en el ámbito del Arte Abstracto, en donde los aspectos relevantes no involucran referencia a figuras humanas ni representaciones materiales. En esa línea, una posible continuación del primer diálogo es la siguiente:

Pedro: Mujer joven leyendo una carta es equilibrada, como todas las pinturas de Vermeer.

Tomás: No, no diría eso. Para mí una obra equilibrada es Broadway Boogie-Woogie, de Mondrian. Lo que quiero decir 
es que Mujer joven leyendo una carta no es equilibrada en el mismo sentido en que Broadway Boogie-Woogie lo es.

Lo que parece estar en juego en esta réplica es la idea de que la manera como las obras abstractas manifiestan el equilibrio es distinta de la manera como lo hacen las obras barrocas, i.e., si bien podríamos suponer que los aspectos relevantes a tomar en cuenta son los mismos (disposición de los objetos en el plano, paleta de colores y distribución de la luz, tamaños comparativos), los objetos cuya disposición y tamaño cuentan no son figuras humanas ni representaciones de objetos materiales (con sus respectivas connotaciones), y la luz no se difumina a través de técnicas como el claroscuro.

En consecuencia, discusiones como las anteriores no revelan la existencia de desacuerdos sin falta sino que a ellas subyace una diferencia de significado, lo que acerca esas discusiones a una disputa meramente verbal. Se trata de algo análogo a lo que ocurre en el siguiente diálogo:

Martín (argentino): ¡Este ananá es delicioso!

Ximena (colombiana): No coincido: ¡esto no es un ananá sino una piña!

Lo que Martín llama 'ananá' en el idiolecto del Río de la Plata es lo que Ximena llama 'piña' en el idiolecto de Bogotá. De la misma manera, lo que Pedro llama 'equilibrio' en el ámbito del Barroco Holandés es lo que Tomás llama 'equilibrio’ en el ámbito del arte contemporáneo. Así como Ximena no está diciendo estrictamente que el ananá no es delicioso sino que esa fruta no es algo que ella llamaría 'ananá', Tomás tampoco está diciendo estrictamente que Mujer joven leyendo una carta no es equilibrada sino que esa obra no es algo que él llamaría 'equilibrado', en el sentido de que no ejemplifica ningún concepto del canon del Arte Abstracto que él suscribe, tiene en mente, o en el que le interesa concentrarse. En síntesis, Desacuerdo 4 y Desacuerdo 3 involucran un cambio de significado, dado que 'equilibrada' no tiene el mismo significado para Pedro, Tomás y Simón: entre Pedro y Tomás, hay una diferencia en la propiedad multidimensional representada, mientras que entre Pedro y Simón la diferencia está en la valencia; por lo tanto, no constituyen ejemplos de desacuerdo (a fortiori, tampoco de desacuerdo sin falta) respecto del grado de equilibrio presente en el cuadro de Vermeer o de su evaluación sino algo semejante a un malentendido. Una condición para que dos personas desacuerden sobre un tema es que utilicen las mismas palabras para referirse a las mismas cosas; es decir, que los significados de las palabras se mantengan fijos a lo largo de la discusión.

Alternativamente, bajo la interpretación menos caritativa según la cual Tomás y Simón no están invocando un canon artístico distinto, el diagnóstico debería ser que ambos están equivocados: (2) debe ser evaluada desde la perspectiva canónica del Barroco Holandés, dado que 'equilibrada' es un predicado teórico cuya aplicación correcta requiere la adopción del estándar estético canónico que caracteriza a esa corriente artística. Quien adopte esa perspectiva, al juzgar (2) y atribuirle una valencia positiva, estará haciendo una afirmación verdadera y evaluativamente correcta $-\mathrm{y}$ si, en cambio, la rechaza o cambia su carga evaluativa, su negación será falsa o evaluativamente incorrecta (o ambas cosas), relativa-mente a la perspectiva canónica correspondiente.

\section{CONCLUSIÓN}

En este ensayo, he defendido la tesis de que los predicados estéticos densos, tales como 'perturbador', 'sombrío', 'equilibrado', son sensi- 
bles a la apreciación, en el mismo sentido en que lo son los predicados de gusto, y, por lo tanto, pueden ser analizados en el marco del relativismo de la verdad. La razón principal que he presentado en favor de esta tesis es que se trata de predicados cuyo uso requiere la aplicación de un estándar estético - entendido como un criterio, por un lado, para seleccionar, medir y ponderar comparativamente distintos dimensiones de una obra de arte y, por otro, para evaluar globalmente esa condición, esto es, determinar si la aplicación del predicado constituye un mérito o un demérito para la obra en juego. Además, he sostenido que los predicados estéticos pueden clasificarse en dos grupos, experienciales y teóricos, según el tipo de estándar estético involucrado en su aplicación. Los experienciales, como 'perturbador', involucran estándares constituidos por estados psicológicos contingentes y aleatorios, característicos de perspectivas estéticas idiosincráticas; los teóricos, como 'equilibrado', involucran la adopción de estándares estables, determinados por perspectivas estéticas canónicas. Esta distinción ha sido utilizada para explicar una falta de asimetría entre unos y otros: el uso de predicados experienciales puede dar lugar a desacuerdos estéticos sin falta, semejantes a los desacuerdos sin falta generados por los predicados de gusto, pero eso no ocurre con los predicados teóricos.

\section{REFERENCIAS}

BlaCKBuRN, Simon (1992), “Through Thick and Thin”, Proceedings of the Aristotelian Society, v. suplementario 66: 285-299.

GibBARD, Alan (1990), Wise Choices, Apt Feelings (Oxford: Oxford University Press).

GlanzberG, Michael (2007), "Context, Content, and Relativism", Philosophical Studies: an Inter-national Journal for Philosophy in the Analytic Tradition, 136, 1: 1-29.

(2009), "Not All Contextual Parameters are Alike" (ms.).
HURKA, Thomas \& ElsTein, Daniel (2009), "From Thick to Thin: Two Moral Reduction Plans", Canadian Journal of Philosophy, 39, 4: 515-536.

KAPLAN, David (1999), "The Meaning of Ouch and Oops: Explorations in the Theory of Mean-ing as Use" (ms.).

KENNEDY, Christopher (2007), "Vagueness and Grammar: the Semantics of Relative and Absolute Gradable Adjectives", Linguistics and Philosophy, 30, 1: 1-45.

KenNedy, Christopher \& MCNALLy, Louise (2005), "Scale Structure, Degree Modification, and the Semantics of Gradable Predicates", Language, 81, 2: 345-38.

KirChIN, Simon (2013), Thick Concepts (Oxford: Oxford University Press). (2017), Thick Evaluation (Oxford: Oxford University Press).

Lasersohn, Peter (2005), "Context Dependence, Disagreement, and Predicates of Personal Taste", Linguistics and Philosophy, 28: 643-686.

MacFarlane, John (2007), "Relativism and Disagreement", Philosophical Studies, 132: 17-31.

(2014), Assessment Sensitivity: Relative Truth and Its Applications (Oxford: Clarendon Press).

MaCNALly, Louise \& STOJANOvic, Isidora, “Aesthetic Adjectives" (2017), en Young (2017: 17-37).

MCCREADY, Eric (2010), "Varieties of Conventional Implicatures”, Semantics and Pragmatics, 3: 1-57.

POTTS, Christopher (2005), The Logic of Conventional Implicature (Oxford: Oxford University Press).

PREDELLI, Stefano (2013), Meaning without Truth (Oxford: Oxford University Press).

SCHAFER, Karl (2011), “Faultless Disagreement and Aesthetic Realism”, Philosophy and Phenomenological Research, 82, 2: 265-286.

Sibley, Frank (1959), "Aesthetic Concepts", Philosophical Review, 68, 4: $421-50$.

Stevenson, Charles (1944), Ethics and Language (New Haven: Yale University Press). 
StojANovic, Isidora (2016), "Expressing Aesthetic Judgments in Context", Inquiry, 59, 6: 663-685.

VÄYRYNEN, Pekka (2011), “Thick Concepts and Variability”, Philosophers' Imprint, 11, 1: 1-17.

Young, James O. (comp.) (2017), The Semantics of Aesthetic Judgment (Oxford: Oxford University Press 